\title{
Gluten-Free Bread: A Better Dietary Option?
}

\section{Sunandita Ghosh*}

University of Leeds, Department of Food Science and Nutrition, Leeds, UK

*Corresponding Author: Sunandita Ghosh, MSc Food Science, University of Leeds, Department of Food Science and Nutrition, Leeds, UK, E-mail: sunandita.ghosh11@gmail.com

Received date: March 02, 2016; Accepted date: March 27, 2017; Published date: March 31, 2017

Copyright: (c) 2017 Ghosh S. This is an open-access article distributed under the terms of the Creative Commons Attribution License, which permits unrestricted use, distribution, and reproduction in any medium, provided the original author and source are credited.

\section{Introduction}

Bread plays an important role in the UK diet and gluten, a protein found in grains such as wheat, barley, rye, and oats is considered an important constituent for both bread-making and as a source of dietary protein. During dough-making, gluten forms a protein network that imparts unique viscoelastic properties that give the characteristic foam structure to the dough [1].

\section{Summary}

Gluten in the diet, however, is not an option for some. Celiac disease is an autoimmune disorder that affects approximately 1 in 100 people in the UK [2]. If celiac patients consume gluten-containing products in the diet, they experience an intestinal mucosal inflammation. This is because the immune system recognizes the gluten products in the intestine as foreign bodies causing it to also attack the healthy tissues which cause the inflammation. The inflammation and damage to the intestinal walls can lead to the inability to absorb the required levels of calcium, iron, folic acid and fat soluble vitamins.

Deficiencies in these elements can lead to conditions such as osteoporosis (weakened bones) and anemia in patients suffering with celiac disease [3]. A Gluten-free diet is advisable for people with celiac disease, wheat allergies and non-celiac gluten sensitivity. Wheat allergy and non-celiac gluten sensitivity is different from celiac disease. In wheat allergy the immune system can respond to any of the proteins present in wheat, hence wheat should be avoided altogether.

Non-celiac gluten sensitivity is neither an autoimmune disorder nor an allergic response; it is a stress response due to gluten intake leading to intestinal and extra intestinal symptoms. Nowadays, even glutentolerant customers are shifting to gluten-free diets they perceive it to be healthier and more effective in weight reduction [4]. Hence, this misconception about gluten free products being healthy and along with increased diagnosis and awareness for gluten-intolerance, has led to the demand for gluten-free bread to rise greatly.

The increase in the consumption of gluten-free bread by those that are tolerant to gluten is largely due to a lack of informed knowledge on the product. A common perception is that a gluten-free diet helps in reducing obesity, has better digestive and nutritional attributes and is of higher quality $[4,5]$. Researchers have suggested it might be a better option for consumers to opt for gluten-free bread due to its lower salt content.
However, gluten-free products are starch-based food with lower protein levels and a higher fat content [6] and lead to an unbalanced diet. This is a major cause of concern; especially for people with gluten sensitivity and no alternative replacement. Though a diet with low salt content is desirable, the bread consumed should also have certain nutritional attributes as bread is widely consumed and is a vital source of carbohydrates, proteins, vitamins and minerals [7].

\section{Conclusion}

Further studies are required to enhance the nutritional qualities of gluten-free bread and other products so that consumers with gluten sensitivity, and those who choose a gluten-free diet, are provided with the same nutritional quality of bread. Efforts are now being made worldwide to also reduce the salt content in regular bread so that consumers without gluten sensitivity have the option of bread with reduced salt content without having to switch to gluten-free bread. With the further development of non-gluten containing bread, same health benefits are expected for all.

\section{References}

1. Buresova I, Stanislav K, Petra D, Tomáš S (2014) The relationship between rheological characteristics of gluten-free dough and the quality of biologically leavened bread. J Cereal Sci 60: 271-275.

2. Coeliac disease (2016) Health A-Z, NHS Choices, UK.

3. Krupa-Kozak U, Altamirano-Fortoul R, Wronkowska M, Rosell CM (2012) Breadmaking performance and technological characteristic of gluten-free bread with inulin supplemented with calcium salts. Eur Food Res Technol 235: 545-554.

4. Miranda J, Lasa A, Bustamante MA, Churruca I, Simon E (2014) Nutritional Differences Between a Gluten-free Diet and a Diet Containing Equivalent Products with Gluten. Plant Foods Hum Nutr 69: 182-187.

5. Sharma GM, Pereira M, Williams KM (2015) Gluten detection in foods available in the United States - A market survey. Food Chem 169:120-126.

6. Segura ME, Rosell CM (2011) Chemical Composition and Starch Digestibility of Different Gluten-free Breads. Plant Foods Hum Nutr 66: 224-230.

7. Quilez J, Salas-Salvado J (2012) Salt in bread in Europe: potential benefits of reduction. Nutr Rev 70: 666-678. 